\title{
On Some Properties of Functions of Generalized Variation
}

\author{
By
}

\section{Maligranda and W. Orlicz, Poznań}

(Received 20 May 1986)

Abstract. We show that the space $V_{\Phi}^{*}$ generated by a $\varphi$-variation (or $\varphi$-variation of Riesz, or $\lambda$-variation of Waterman) forms a commutative Banach algebra with respect to the pointwise multiplication under the appropriate choice of norms.

1. By a $\varphi$-function we mean here a non-decreasing continuous function $\varphi:[0, \infty) \rightarrow[0, \infty)$ such that $\varphi(u)=0$ if and only if $u=0$ and $\varphi(u) \rightarrow \infty$ as $u \rightarrow \infty$.

Throughout this paper $X$ will denote a vector space of real valued functions $x$ defined in a finite interval $[a, b]$, for which $x(a)=0$. Let $x \in X$. For a $\varphi$-function $\phi$ and for a partition $\pi: a=t_{0}<t_{1}<\ldots<$ $<t_{n}=b$ of $[a, b]$ denote

$$
\sigma_{\varphi}(x, \pi)=\sum_{k=1}^{n} \varphi\left(\left|x\left(t_{k}\right)-x\left(t_{k-1}\right)\right|\right) .
$$

The number

$$
v_{\varphi}(x)=\sup _{\pi} \sigma_{\varphi}(x, \pi),
$$

where supremum is taken over all partitions $\pi$ of $[a, b]$, is called the $\varphi$ variation of $x$ on $[a, b]$.

When $v_{\varphi}(x)<\infty$ the function $x$ is said to have finite (or bounded) $\varphi$-variation or be of $\varphi-\mathrm{BV}$. An element $x \in X$ is said to satisfy condition B.1 ([7], p. 50) if $v_{\varphi}(\lambda x) \rightarrow 0$ as $\lambda \rightarrow 0$. In the vector space $V_{\varphi}^{0}=\{x \in X: x$ satisfies B. 1$\}$ we may define a complete $F$-norm by

$$
|x|_{\varphi}^{v}=\inf \left\{\varepsilon>0: v_{\varphi}(x / \varepsilon) \leqslant \varepsilon\right\} .
$$

By $V_{\varphi}$ we shall denote the class of all functions of finite $\varphi$-variation and by $V_{\varphi}^{*}$ the vector space of all functions $x \in X$ such that $\lambda x \in V_{\varphi}$ for some 
$\lambda>0$. Clearly, $V_{\varphi} \subset V_{\varphi}^{*}$ and $V_{\varphi}{ }^{0} \subset V_{\varphi}^{*}$. Let us define a homogeneous functional (pseudonorm) $\|\cdot\|_{\varphi}^{0}$ on $V_{\varphi}^{*}$ by

$$
\|x\|_{\varphi}^{v}=\inf \left\{\varepsilon>0: v_{\varphi}(x / \varepsilon) \leqslant 1\right\}, \quad \inf \emptyset=\infty .
$$

It is not a norm, in general, because the triangle inequality does not hold. When $\varphi$ is a $\varphi$-function of the form $\varphi(u)=\psi\left(u^{s}\right), 0<s \leqslant 1$, where $\psi$ is a convex $\phi$-function, then in $V_{\varphi}^{*}=V_{\varphi}{ }^{0}$ we may define an $s$ homogeneous norm (homogeneous when $s=1$, i.e. when $\varphi$ is convex) by

$$
\|x\|_{s \varphi}^{v}=\inf \left\{\varepsilon>0: v_{\varphi}\left(x / \varepsilon^{1 / s}\right) \leqslant 1\right\},
$$

equivalent to $|\cdot|_{\varphi}^{v}$ (cf. [4], p. 85). It is easy to see that $\left(\|x\|_{s \varphi}^{v}\right)^{1 / s}=$ $=\|x\|_{\varphi}^{v}$. If $\varphi(u)=u^{p}(p>0)$ we write a letter $p$ instead of $\varphi$ in the above notation.

2. Let $\varphi$ and $\psi$ are the $\phi$-functions. The inverse of $\psi$ is defined on $[0, \infty)$ by

$$
\psi^{-1}(u)=\inf \{s \geqslant 0: \psi(s)>u\} .
$$

If there exists a positive constant $c$ such that

then

$$
\varphi(u) \leqslant \psi(u) \text { for } u \in[0, c]
$$

$$
\|x\|_{\varphi}^{v} \leqslant \max \left(1, \psi^{-1}(1) / c\right)\|x\|_{\psi}^{v} .
$$

Proof. We assume that $\lambda=\|x\|_{\psi}^{v}<\infty$. For any partition $\pi: a=t_{0}<t_{1}<\ldots<t_{n}=b$ we have $\sigma_{\psi}(x / \lambda, \pi) \leqslant 1$, i.e. $\psi\left(\left|x\left(t_{k}\right)-x\left(t_{k-1}\right)\right| / \lambda\right) \leqslant 1$ for $k=1,2 \ldots, n$.

Consequently,

$$
\begin{gathered}
\left|x\left(t_{k}\right)-x\left(t_{k-1}\right)\right| / \lambda \leqslant \psi^{-1}\left[\psi\left(\left|x\left(t_{k}\right)-x\left(t_{k-1}\right)\right| / \lambda\right)\right] \leqslant \\
\leqslant \psi^{-1}(1) \leqslant c \max \left(1, \psi^{-1}(1) / c\right) .
\end{gathered}
$$

By the assumption,

$$
\begin{gathered}
\varphi\left(\frac{\left|x\left(t_{k}\right)-x\left(t_{k-1}\right)\right|}{\lambda \max \left(1, \psi^{-1}(1) / c\right)}\right) \leqslant \psi\left(\frac{\left|x\left(t_{k}\right)-x\left(t_{k-1}\right)\right|}{\lambda \max \left(1, \psi^{-1}(1) / c\right)}\right) \leqslant \\
\leqslant \psi\left(\left|x\left(t_{k}\right)-x\left(t_{k-1}\right)\right| / \lambda\right) .
\end{gathered}
$$

Therefore, the previous estimate ensures that

$$
\sigma_{\varphi}\left(x /\left[\lambda \max \left(1, \psi^{-1}(1) / c\right], \pi\right) \leqslant \sigma_{\psi}(x / \lambda, \pi) \leqslant 1 .\right.
$$


Hence

$$
\|x\|_{\varphi}^{v} \leqslant \lambda \max \left(1, \psi^{-1}(1) / c\right)=\|x\|_{\psi}^{v} \max \left(1, \psi^{-1}(1) / c\right) .
$$

In particular, taking $\varphi(u)=u^{p}$ and $\psi(u)=u^{q}, 0<p<q$ we have the following inequality

$$
v_{q}(x)^{1 / q} \leqslant v_{p}(x)^{1 / p} .
$$

3. In this and further parts normed subspaces $\mathfrak{X}$ of $X$ will be considered such that their elements are bounded functions, and which are commutative Banach algebras with respect to pointwise multiplication. The question here is to find in $(\mathfrak{X},\|\cdot\|)$ a norm $|\cdot|$ equivalent to $\|\cdot\|$ such that the multiplication and the norm $|\cdot|$ are linked by the inequality

$$
|x y| \leqslant|x||y| \text { for all } x, y \in \mathfrak{X} .
$$

3.1. Lemma. Let $a_{0}=b_{0}=0$ and $a_{k}, b_{k}, k=1,2, \ldots$, $n$ are any real numbers. For $0<p \leqslant 1$ the following inequality holds:

$$
\sum_{k=1}^{n}\left|a_{k} b_{k}-a_{k-1} b_{k-1}\right|^{p} \leqslant\left(\sum_{k=1}^{n}\left|a_{k}-a_{k-1}\right|^{p}\right)\left(\sum_{k=1}^{n}\left|b_{k}-b_{k-1}\right|^{p}\right) \text {. }
$$
Then

Proof (by induction). For $n=1$ inequality (4) is true. Let $n=2$.

$$
\begin{gathered}
\left|a_{2} b_{2}-a_{1} b_{1}\right|^{p}=\left|\left(a_{2}-a_{1}\right)\left(b_{2}-b_{1}\right)+a_{1}\left(b_{2}-b_{1}\right)+b_{1}\left(a_{2}-a_{1}\right)\right|^{p} \leqslant \\
\leqslant\left|a_{2}-a_{1}\right|^{p}\left|b_{2}-b_{1}\right|^{p}+\left|a_{1}\right|^{p}\left|b_{2}-b_{1}\right|^{p}+\left|b_{1}\right|^{p}\left|a_{2}-a_{1}\right|^{p}
\end{gathered}
$$

and, consequently,

$$
\left|a_{2} b_{2}-a_{1} b_{1}\right|^{p}+\left|a_{1} b_{1}\right|^{p} \leqslant\left(\left|a_{2}-a_{1}\right|^{p}+\left|a_{1}\right|^{p}\right)\left(\left|b_{2}-b_{1}\right|^{p}+\left|b_{1}\right|^{p}\right) .
$$

Now, suppose that inequality (4) is true for $n-1$. Using the equality $a_{n} b_{n}-a_{n-1} b_{n-1}=\left(a_{n}-a_{n-1}\right)\left(b_{n}-b_{n-1}\right)+a_{n-1}\left(b_{n}-b_{n-1}\right)+$ $+b_{n-1}\left(a_{n}-a_{n-1}\right)$ we have

$$
\begin{aligned}
& \sum_{k=1}^{n}\left|a_{k} b_{k}-a_{k-1} b_{k-1}\right|^{p} \leqslant\left(\sum_{k=1}^{n-1}\left|a_{k}-a_{k-1}\right|^{p}\right)\left(\sum_{k=1}^{n-1}\left|b_{k}-b_{k-1}\right|^{p}\right)+ \\
+ & \left|a_{n}-a_{n-1}\right|^{p}\left|b_{n}-b_{n-1}\right|^{p}+\left|a_{n-1}\right|^{p}\left|b_{n}-b_{n-1}\right|^{p}+\left|a_{n}-a_{n-1}\right|^{p}\left|b_{n-1}\right|^{p} .
\end{aligned}
$$

Since $\left|a_{n-1}\right|^{p}=\left|\sum_{k=1}^{n-1}\left(a_{k}-a_{k-1}\right)\right|^{p} \leqslant\left(\sum_{k=1}^{n-1}\left|a_{k}-a_{k-1}\right|\right)^{p} \leqslant \sum_{k=1}^{n-1}\left|a_{k}-a_{k-1}\right|^{p}$ 
and, similarly, $\left|b_{n-1}\right|^{p} \leqslant \sum_{k=1}^{n-1}\left|b_{k}-b_{k-1}\right|^{p}$ it follows that

$$
\begin{gathered}
\sum_{k=1}^{n}\left|a_{k} b_{k}-a_{k-1} b_{k-1}\right|^{p} \leqslant \\
\leqslant\left(\sum_{k=1}^{n-1}\left|a_{k}-a_{k-1}\right|^{p}+\left|a_{n}-a_{n-1}\right|^{p}\right)\left(\sum_{k=1}^{n-1}\left|b_{k}-b_{k-1}\right|^{p}+\left|b_{n}-b_{n-1}\right|^{p}\right) .
\end{gathered}
$$

Therefore, inequality (4) is true for all natural numbers $n$.

3.2. Theorem 1. If $0<p \leqslant 1$ then

$$
v_{p}(x y) \leqslant v_{p}(x) v_{p}(y) \text { for } x, y \in V_{p} .
$$

Proof. For a given partition $\pi: a=t_{0}<t_{1}<\ldots<t_{n}=b$ let $a_{k}=x\left(t_{k}\right)$ and $b_{k}=y\left(t_{k}\right)$. Using Lemma 3.1 we have

$$
\sigma_{p}(x y, \pi) \leqslant \sigma_{p}(x, \pi) \sigma_{p}(y, \pi) \leqslant v_{p}(x) v_{p}(y) .
$$

Since $\pi$ is an arbitrary partition of $[a, b]$, we get inequality (5).

Note that the proof of Lemma 3.1 can be repeated without change also for any subadditive and submultiplicative $\varphi$-function $\varphi$. This means that for such $\varphi$ the inequality of type (5) holds.

If $p=1$ this theorem with another proof can be found in [1], [8].

Inequality (5) is not satisfied, in general, for $p>1$. To show this we define a function

$$
x_{\varepsilon}(t)= \begin{cases}0 & \text { for } 0 \leqslant t \leqslant 1 / 3, \\ 1+\varepsilon & \text { for } 1 / 3<t<1 / 2, \quad(\varepsilon>0) \\ 1 & \text { for } 1 / 2 \leqslant t \leqslant 1 .\end{cases}
$$

Then $v_{p}\left(x_{\varepsilon}\right)=(1+\varepsilon)^{p}+\varepsilon^{p}$ and $v_{p}\left(x_{\varepsilon}^{2}\right)=(1+\varepsilon)^{2 p}+\left(2 \varepsilon+\varepsilon^{2}\right)^{p}$. If inequality (5) holds then $v_{p}\left(x_{\varepsilon}^{2}\right) \leqslant v_{p}\left(x_{\varepsilon}\right)^{2}$ for any $\varepsilon>0$. This means that $(1+\varepsilon)^{2 p}+\left(2 \varepsilon+\varepsilon^{2}\right)^{p} \leqslant\left((1+\varepsilon)^{p}+\varepsilon^{p}\right)^{2}$ and, consequently, $(2+\varepsilon)^{p} \leqslant 2(1+\varepsilon)^{p}+\varepsilon^{p}$. Taking $\varepsilon \rightarrow 0$ we get $2^{p} \leqslant 2$, i.e. $p \leqslant 1$.

Theorem 1 means that $v_{1}\left(|x|^{n}\right) \leqslant v_{1}(x)^{n}$ for any natural number $n$. We show below that this inequality is also true if in the place of $\varphi(u)=u^{n}$ we take any convex $\varphi$-function.

3.3. If $\varphi$ is a convex $\varphi$-function and $x \in V_{1}$, then $\varphi(|x|) \in V_{1}$ and

$$
v_{1}(\varphi(|x|)) \leqslant \varphi\left(v_{1}(|x|)\right) .
$$


Proof. It is sufficient to prove that if $a_{0}=0$ and $a_{k} \geqslant 0$ for $k=1,2, \ldots, n$, then

$$
\sum_{k=1}^{n}\left|\varphi\left(a_{k}\right)-\varphi\left(a_{k-1}\right)\right| \leqslant \varphi\left(\sum_{k=1}^{n}\left|a_{k}-a_{k-1}\right|\right) .
$$

We will prove inequality (7), by induction using the integral representation of a convex $\varphi$-function $\varphi$, that is, $\varphi(u)=\int_{0}^{u} p(s) d s$, where $p$ is a
non-negative non-decreasing function.

For $n=1$ inequality (7) is true. Let $n=2$. If $a_{1}<a_{2}$ then $\left|\varphi\left(a_{2}\right)-\varphi\left(a_{1}\right)\right|+\varphi\left(a_{1}\right)=\varphi\left(a_{2}\right)=\varphi\left(\left|a_{2}-a_{1}\right|+a_{1}\right)$ and if $a_{1}>a_{2}$ then

$$
\begin{aligned}
\mid \varphi\left(a_{2}\right) & -\varphi\left(a_{1}\right) \mid+\varphi\left(a_{1}\right)=2 \varphi\left(a_{1}\right)-\varphi\left(a_{2}\right)=\varphi\left(a_{1}\right)+\int_{a_{2}}^{a_{1}} p(s) \\
& \leqslant \varphi\left(a_{1}\right)+p\left(a_{1}\right)\left(a_{1}-a_{2}\right) \leqslant \varphi\left(a_{1}\right)+\int_{a_{1}}^{2 a_{1}-a_{2}} p(s) d s= \\
& =\varphi\left(2 a_{1}-a_{2}\right)=\varphi\left(\left|a_{2}-a_{1}\right|+a_{1}\right) .
\end{aligned}
$$

Now, assume that inequality (7) is true for $n-1$. Then

$$
\sum_{k=1}^{n}\left|\varphi\left(a_{k}\right)-\varphi\left(a_{k-1}\right)\right| \leqslant \varphi\left(\sum_{k=1}^{n-1}\left|a_{k}-a_{k-1}\right|\right)+\left|\varphi\left(a_{n}\right)-\varphi\left(a_{n-1}\right)\right| .
$$

We are obliged to show that

$$
\varphi(A)+\left|\varphi\left(a_{n}\right)-\varphi\left(a_{n-1}\right)\right| \leqslant \varphi\left(A+\left|a_{n}-a_{n-1}\right|\right),
$$

where $A=\sum_{k=1}^{n-1}\left|a_{k}-a_{k-1}\right|$.

Let us consider three cases (always $a_{n-1} \leqslant A$ ):

$1^{\circ}$. Let $a_{n} \leqslant a_{n-1}$. Then

$$
\begin{aligned}
& \varphi(A)-\varphi\left(a_{n}\right)=\int_{a_{n}}^{A} p(s) d s=\int_{a_{n}}^{a_{n-1}} p(s) d s+\int_{a_{n-1}}^{A} p(s) d s \leqslant \\
& \leqslant p\left(a_{n-1}\right)\left(a_{n-1}-a_{n}\right)+\int_{a_{n-1}}^{A} p(s) d s \leqslant p(A)\left(a_{n-1}-a_{n}\right)+\int_{a_{n-1}}^{A} p(s) d s \leqslant \\
& \leqslant \int_{A}^{A+a_{n-1}-a_{n}} p(s) d s+\int_{a_{n-1}}^{A} p(s) d s=
\end{aligned}
$$




$$
=\int_{a_{n-1}}^{A+a_{n-1}-a_{n}} p(s) d s=\varphi\left(A+a_{n-1}-a_{n}\right)-\varphi\left(a_{n-1}\right) .
$$

$2^{\circ}$. Let $a_{n-1} \leqslant A \leqslant a_{n}$. Then

$$
\begin{gathered}
\varphi(A)-\varphi\left(a_{n-1}\right)=\int_{a_{n-1}}^{A} p(s) d s \leqslant p(A)\left(A-a_{n-1}\right) \leqslant p\left(a_{n}\right)\left(A-a_{n-1}\right) \leqslant \\
\leqslant \int_{a_{n}}^{A+a_{n}-a_{n-1}} p(s) d s=\varphi\left(A+a_{n}-a_{n-1}\right)-\varphi\left(a_{n}\right) .
\end{gathered}
$$

$3^{\circ}$. Let $a_{n-1} \leqslant a_{n} \leqslant A$. Then

$$
\begin{aligned}
& \varphi(A)-\varphi\left(a_{n-1}\right)=\int_{a_{n-1}}^{A} p(s) d s=\int_{a_{n-1}}^{a_{n}} p(s) d s+\int_{a_{n}}^{A} p(s) d s \leqslant \\
& \leqslant p\left(a_{n}\right)\left(a_{n}-a_{n-1}\right)+\int_{a_{n}}^{A} p(s) d s \leqslant p(A)\left(a_{n}-a_{n-1}\right)+\int_{a_{n}}^{A} p(s) d s \leqslant
\end{aligned}
$$

$$
\begin{aligned}
& \leqslant \int_{A}^{A+a_{n}-a_{n-1}} p(s) d s+\int_{a_{n}}^{A} p(s) d s=\int_{a_{n}}^{A+a_{n}-a_{n-1}} p(s) d s= \\
& =\varphi\left(A+a_{n}-a_{n-1}\right)-\varphi\left(a_{n}\right) .
\end{aligned}
$$

This proves our claim.

3.4. Now, we apply inequalities (6) or (5) if $p=1$, to prove of the inequality of Opial type (for $p=1$ we have the well known Opial inequality; cf. [5]).

Let $x$ be an absolutely continuous function on $[a, b]$ with $x(a)=0$ and let $p \geqslant 0$. Then

$$
\int_{a}^{b}|x(t)|^{p}\left|x^{\prime}(t)\right| d t \leqslant(b-a)^{p}(p+1)^{-1} \int_{a}^{b}\left|x^{\prime}(t)\right|^{p+1} d t .
$$

Namely, if $\varphi(u)=u^{p+1}$ then $\varphi(|x|)$ is absolutely continuous on $[a, b]$ and

$$
(p+1) \int_{a}^{b}|x(t)|^{p}\left|x^{\prime}(t)\right| d t=\int_{a}^{b}\left|\varphi(|x|)^{\prime}(t)\right| d t=v_{1}(\varphi(|x|)) .
$$

Consequently, from (6) or (5) if $p=1$ and from the Hölder inequality we obtain

$$
v_{1}(\varphi(|x|)) \leqslant \varphi\left(v_{1}(x)\right)=\left(\int_{a}^{b}\left|x^{\prime}(t)\right| d t\right)^{p+1} \leqslant \int_{a}^{b}\left|x^{\prime}(t)\right|^{p+1} d t(b-a)^{p}
$$


from which the conclusion follows. Using the above considerations it is not difficult to prove a more general inequality of Opial type from [2].

4. In this and further parts we give some examples of typical function spaces with finite variation which become normed Banach algebras with pointwise multiplication of functions under the appropriate choice of norms. The idea of the proof is based on the following simple lemma.

4.1. Let $(\mathfrak{X},\|\cdot\|)$ be a Banach subspace of $X$ whose elements are bounded functions. Let us assume that $x y \in \mathfrak{X}$ and

$$
\|x y\| \leqslant\|x\|_{\infty}\|y\|+\|x\|\|y\|_{\infty} \text { for any } x, y \in \mathfrak{X} .
$$

Then the space $\mathfrak{X}$ equipped with the norm

$$
|x|=\|x\|_{\infty}+\|x\|
$$

is a normed Banach algebra. If $\left\|x_{n}\right\| \rightarrow 0$ implies $\left\|x_{n}\right\|_{\infty} \rightarrow 0$ then the norms $|\cdot|$ and $\|\cdot\|$ are equivalent. Moreover, if $\|x\|_{\infty} \leqslant c\|x\|$ for $x \in \mathfrak{X}$, then $\mathfrak{X}$ equipped with the norm

$$
|x|=2 c\|x\|
$$

is also a normed Banach algebra.

It is sufficient to prove inequality (3). For the first norm we have

$$
\begin{aligned}
& |x y|=\|x y\|_{\infty}+\|x y\| \leqslant\|x\|_{\infty}\|y\|_{\infty}+\|x y\| \leqslant\|x\|_{\infty}\|y\|_{\infty}+ \\
& +\|x\|_{\infty}\|y\|+\|x\|\|y\|_{\infty} \leqslant\left(\|x\|_{\infty}+\|x\|\right)\left(\|y\|_{\infty}+\|y\|\right)=|x \| y|,
\end{aligned}
$$

and for the second one

$$
\begin{gathered}
|x y|=2 c\|x y\| \leqslant 2 c\|x\|_{\infty}\|y\|+2 c\|x\|\|y\|_{\infty}= \\
=\|x\|_{\infty}|y|+|x|\|y\|_{\infty} \leqslant c\|x\||y|+c|x|\|y\|=|x||y| .
\end{gathered}
$$

4.2. Theorem 2. Let $\varphi$ be a convex $\varphi$-function. Then $V_{\varphi}^{*}$ with the norm $\|\cdot\|_{\varphi}^{v}$ is a Banach space and with the norms $|x|=\|x\|_{\infty}+\|x\|_{p}^{v}$ or $|x|=2 \varphi^{-1}(1)\|x\|_{p}^{v}$ is a normed Banach algebra.

Proof. Since $\varphi(|x(t)|) \leqslant v_{\varphi}(x)$ for any $t \in[a, b]$ it follows that

$$
\|x\|_{\infty} \leqslant \varphi^{-1}(1)\|x\|_{\varphi}^{v} \text { for } x \in V_{\varphi}^{*} \text {. }
$$


The assertion would be true if we verify that inequality (8) holds. Let $x, y \in V_{\varphi}^{*}$. Consider a partition $\pi: a=t_{0}<t_{1}<\ldots<t_{n}=b$. Putting $\|x\|_{\infty}=\lambda_{1},\|y\|_{\infty}=\lambda_{2}$ and $\lambda_{2}\|x\|_{\varphi}^{v}+\lambda_{1}\|y\|_{\varphi}^{v}=\lambda$, we have

$$
\begin{gathered}
s_{k}=\left|x\left(t_{k}\right) y\left(t_{k}\right)-x\left(t_{k-1}\right) y\left(t_{k-1}\right)\right|=\mid\left[x\left(t_{k}\right)-x\left(t_{k-1}\right)\right] y\left(t_{k}\right)+ \\
+x\left(t_{k-1}\right)\left[y\left(t_{k}\right)-y\left(t_{k-1}\right)\right]\left|\leqslant \lambda_{2}\right| x\left(t_{k}\right)-x\left(t_{k-1}\right)\left|+\lambda_{1}\right| y\left(t_{k}\right)-y\left(t_{k-1}\right) \mid
\end{gathered}
$$

and by convexity of $\varphi$

$$
\begin{aligned}
\varphi\left(\frac{s_{k}}{\lambda}\right) & \leqslant \varphi\left(\frac{\left|x\left(t_{k}\right)-x\left(t_{k-1}\right)\right|}{\|x\|_{\varphi}^{v}} \frac{\lambda_{2}\|x\|_{\varphi}^{v}}{\lambda}+\frac{\left|x\left(t_{k}\right)-y\left(t_{k-1}\right)\right|}{\|y\|_{\varphi}^{v}} \frac{\lambda_{1}\|y\|_{\varphi}^{v}}{\lambda}\right) \leqslant \\
& \leqslant \varphi\left(\left|x\left(t_{k}\right)-x\left(t_{k-1}\right)\right| /\|x\|_{\varphi}^{v}\right) \lambda_{2}\|x\|_{\varphi}^{v} / \lambda+ \\
& +\varphi\left(\left|y\left(t_{k}\right)-y\left(t_{k-1}\right)\right| /\|v\|_{\eta}^{v}\right) \lambda_{1}\|y\|_{\varphi}^{v} / \lambda .
\end{aligned}
$$

Summing over $k$ we obtain

$$
\begin{gathered}
\sigma_{\varphi}(x y / \lambda, \pi) \leqslant \sigma_{\varphi}\left(x /\|x\|_{\varphi}^{v}, \pi\right) \lambda_{2}\|x\|_{\varphi}^{v} / \lambda+\sigma_{\varphi}\left(y /\|y\|_{\varphi}^{v}, \pi\right) \lambda_{1}\|y\|_{\varphi}^{v} / \lambda \leqslant \\
\leqslant \lambda_{2}\|x\|_{\varphi}^{v} / \lambda+\lambda_{1}\|y\|_{\varphi}^{v} / \lambda=1 .
\end{gathered}
$$

Hence, $\|x y\|_{\varphi}^{v} \leqslant \lambda$ and this concludes the proof of the inequality (8).

From Theorem 2, for $\varphi(u)=u^{p}, p \geqslant 1$ and for $x, y \in V_{p}$ we have the following inequalities:

$$
\|x y\|_{\infty}+v_{p}(x y)^{1 / p} \leqslant\left(\|x\|_{\infty}+v_{p}(x)^{1 / p}\right)\left(\|y\|_{\infty}+v_{p}(y)^{1 / p}\right)
$$

and

$$
v_{p}(x y) \leqslant 2^{p} v_{p}(x) v_{p}(y) .
$$

Note that for $p=1$ the last inequalities are essentially weaker than inequality (5).

4.3. Let $F$ denote a real function defined on $R=(-\infty, \infty)$ with the following property:

(A) There exist polynomials $w_{n}(u)=a_{0, n}+a_{1, n} u+\ldots+$ $+a_{k(n) n} u^{k(n)}$, with non-negative coefficients such that $w_{n}(u) \rightarrow F(u)$ for $u \in R$.

Let $F$ be a function with property (A). If $x \in V_{\varphi}^{*}$, where $\varphi$ is a convex $\varphi$-function and $F(0)=0$, then $F(x) \in V_{\varphi}^{*}$ and

$$
|F(x)| \leqslant F(|x|) .
$$


Proof. From Theorem 2 we have

$$
\left|w_{n}(x)\right| \leqslant a_{0, n}+a_{1, n}|x|+\ldots+a_{k(n), n}|x|^{k(n)}=w_{n}(|x|),
$$

and from property (A) of $F$ we have $w_{n}(|x|) \rightarrow F(|x|)$, i.e.

$$
\limsup _{n \rightarrow \infty}\left|w_{n}(x)\right| \leqslant F(|x|) .
$$

Using again property (A) we get $w_{n}(x(t)) \rightarrow F(x(t))$ for $t \in[a, b]$. It follows that the inequalities

$$
\|F(x)\|_{\infty} \leqslant \liminf _{n \rightarrow \infty}\left\|w_{n}(x)\right\|_{\infty} \text { and }\|F(x)\|_{\varphi}^{v} \leqslant \liminf _{n \rightarrow \infty}\left\|w_{n}(x)\right\|_{\varphi}^{v}
$$

hold.

Hence $F(x) \in V_{\varphi}^{*}$ and

$$
|F(x)| \leqslant \liminf _{n \rightarrow \infty}\left|w_{n}(x)\right| \leqslant \limsup _{n \rightarrow \infty}\left|w_{n}(x)\right| \leqslant F(|x|) .
$$

Remark. Property (A) is satisfied if derivatives of $F$ of all orders are non-negative, i.e. $F$ is absolutely monotonic in $R$ in the sense of $\mathrm{S}$. BERnSTEIN. For the connection of property (A) and the class of absolutely monotonic functions (cf. [11], Chapter 4).

5. Let $\varphi$ be a $\varphi$-function and $x \in X$. For a given partition $\pi: a=$ $=t_{0}<t_{1}<\ldots<t_{n}=b$ let

$$
\sigma_{p}^{R}(x, \pi)=\sum_{k=1}^{n} \varphi\left(\frac{\left|x\left(t_{k}\right)-x\left(t_{k-1}\right)\right|}{t_{k}-t_{k-1}}\right)\left(t_{k}-t_{k-1}\right) .
$$

The number

$$
v_{\varphi}^{R}(x)=\sup _{\pi} \sigma_{\varphi}^{R}(x, \pi),
$$

where supremum is taken over all partitions $\pi$, is called the Riesz $\varphi$ variation of $x$. By $R_{\varphi}^{*}$ we shall denote the set of all $x \in X$ such that $v_{\varphi}^{R}(\lambda x)<\infty$ for some $\lambda>0$.

If $\varphi$ is a convex $\varphi$-function, then $R_{\varphi}^{*}$ is a Banach space with respect to the norm

$$
\|x\|_{p}^{R}=\inf \left\{\varepsilon>0: v_{\varphi}^{R}(x / \varepsilon) \leqslant 1\right\},
$$

and for $x \in R_{\varphi}^{*}$

$$
v_{1}(x) \leqslant\left(\varphi(1)^{-1}+b-a\right)\|x\|_{\varphi}^{R} .
$$


In the next we shall need the following condition $\left(\infty_{1}\right)$

$$
\lim _{u \rightarrow \infty} \sup \varphi(u) / u=\infty .
$$

Note that if condition $\left(\infty_{1}\right)$ is not satisfied then $V_{1} \subset R_{\varphi}^{*}$. Indeed, if there exist $r, u_{0}>0$ such that $\varphi(u) \leqslant r u$ for $u \geqslant u_{0}$ then for a given partition $\pi$ the following inequalities hold

$$
\begin{aligned}
\sigma_{\varphi}^{R}(x, \pi) & =\left(\sum_{k \in e_{u_{0}}}+\sum_{k \notin e_{u_{0}}}\right) \varphi\left(\frac{\left|x\left(t_{k}\right)-x\left(t_{k-1}\right)\right|}{t_{k}-t_{k-1}}\right)\left(t_{k}-t_{k-1}\right) \leqslant \\
& \leqslant \sum_{k \in e_{u_{0}}} r\left|x\left(t_{k}\right)-x\left(t_{k-1}\right)\right|+\sum_{k \notin e_{u_{0}}} \varphi\left(u_{0}\right)\left(t_{k}-t_{k-1}\right) \leqslant \\
& \leqslant r \sigma_{1}(x, \pi)+\varphi\left(u_{0}\right)(b-a) \leqslant r v_{1}(x)+\varphi\left(u_{0}\right)(b-a),
\end{aligned}
$$

where $e_{u_{0}}=\left\{k:\left|x\left(t_{k}\right)-x\left(t_{k-1}\right)\right| \geqslant u_{0}\left(t_{k}-t_{k-1}\right)\right\}$. Therefore, $v_{\varphi}^{R}(x) \leqslant$ $\leqslant r v_{1}(x)+r u_{0}(b-a)$, i.e. $V_{1} \subset R_{p}^{*}$. From the above it follows that if $\varphi$ is a convex $\varphi$-function and $\lim _{u \rightarrow \infty} \varphi(u) / u=r<\infty$, then $R_{\varphi}^{*}=V_{1}$ and

$$
\|x\|_{\varphi}^{R} / r \leqslant v_{1}(x) \leqslant\left(\varphi(1)^{-1}+b-a\right)\|x\|_{\varphi}^{R} .
$$

For a convex $\varphi$-function $\varphi$ which satisfies $\left(\infty_{1}\right)$ the following properties of Riesz $\varphi$-variation are known (cf. [3]):

$1^{\circ}$. If $v_{p}^{R}(x)<\infty$, then $x$ is absolutely continuous on $[a, b]$ and $v_{\varphi}^{R}(x)=\int_{a}^{b} \varphi\left(\left|x^{\prime}(t)\right|\right) d t$

$2^{\circ}$. If $x$ is an absolutely continuous function on $[a, b]$ and $\int_{a}^{b} \varphi\left(\left|x^{\prime}(t)\right|\right) d t<\infty$, then $v_{\varphi}^{R}(x)<\infty$;

$$
3^{\circ} .\|x\|_{\varphi}^{R}=\left\|x^{\prime}\right\|_{\varphi}=\inf \left\{\varepsilon>0: \int_{a}^{b} \varphi\left(\left|x^{\prime}(t)\right| / \varepsilon\right) d t \leqslant 1\right\} .
$$

5.1. For a convex $\varphi$-function $\varphi$ which satisfies condition $\left(\infty_{1}\right)$ and for $h=b-a>0$, let $c_{\varphi}(h)=\max \left\{\min \left\{\varphi(1)^{-1}, h^{-1} \varphi\left(h^{-1}\right)^{-1}\right\}\right.$, $\left.h \varphi^{-1}\left(h^{-1}\right)\right\}$. For example, if $\varphi(u)=u^{p}, p>1$, then $c_{\varphi}(h)=h^{1-1 / p}$.

Theorem 3. The space $R_{w}^{*}$ with the norms

$$
|x|=\|x\|_{\infty}+\|x\|_{\varphi}^{R} \text { or }|x|=2 c_{\varphi}(h)\|x\|_{\varphi}^{R}
$$

is a normed Banach algebra. 
Proof. First of all we prove that for $x \in R_{\varphi}^{*}$

$$
\|x\|_{\infty} \leqslant c_{p}(h)\|x\|_{\varphi}^{R} \text {. }
$$

Namely, for a given $x \in X$ let $e=\{s \in[a, b]: h|x(s)| \geqslant s-a\}$. Then from the convexity of $\phi$, we have, for $t \in e$

$$
h|x(t)| \varphi\left(h^{-1}\right) \leqslant \varphi\left(\frac{|x(t)|}{t-a}\right)(t-a) \leqslant v_{p}^{R}(x),
$$

and

$$
h \varphi(1)|x(t)| \leqslant \varphi\left(\frac{h|x(t)|}{t-a}\right)(t-a) \leqslant v_{\varphi}^{R}(h x) .
$$

For $t \in[a, b] \backslash e$ we have

$$
\varphi\left(h^{-1}|x(t)|\right)=\varphi\left(\frac{|x(t)| t-a}{t-a} \frac{t-a}{h}\right) \leqslant \frac{t-a}{h} \varphi\left(\frac{|x(t)|}{t-a}\right) \leqslant h^{-1} v_{\varphi}^{R}(x) .
$$

Consequently,

and

$$
|x(t)| \leqslant \max \left\{h^{-1} \varphi\left(h^{-1}\right)^{-1} v_{\varphi}^{R}(x), h \varphi^{-1}\left(h^{-1} v_{\varphi}^{R}(x)\right)\right\}
$$

$$
|x(t)| \leqslant \max \left\{h^{-1} \varphi(1)^{-1} v_{\varphi}^{R}(h x), \varphi^{-1}\left(h^{-1} v_{\varphi}^{R}(h x)\right)\right\}
$$

for any $t \in[a, b]$. Hence,

and

$$
\|x\|_{\infty} \leqslant \max \left\{h^{-1} \varphi\left(h^{-1}\right)^{-1}, h \varphi^{-1}\left(h^{-1}\right)\right\}\|x\|_{\varphi}^{R}
$$

$$
\|x\|_{\infty} \leqslant \max \left\{\varphi(1)^{-1}, h \varphi^{-1}\left(h^{-1}\right)\right\}\|x\|_{\varphi}^{R}
$$

for $x \in R_{\varphi}^{*}$. Therefore, inequality (14) holds.

The inequality of type (8) can be proved similarly as in the proof of Theorem 2 . However, using properties $1^{\circ}-3^{\circ}$ we give now another simple proof. Indeed, for $x, y \in R_{\varphi}^{*}$ we have

$$
\begin{aligned}
\|x y\|_{\varphi}^{R} & =\left\|(x y)^{\prime}\right\|_{\varphi}=\left\|x y^{\prime}+x^{\prime} y\right\|_{\varphi} \leqslant\left\|x y^{\prime}\right\|_{\varphi}+\left\|x^{\prime} y\right\|_{\varphi} \leqslant \\
& \leqslant\|x\|_{\infty}\left\|y^{\prime}\right\|_{\varphi}+\left\|x^{\prime}\right\|_{\varphi}\|y\|_{\infty}=\|x\|_{\infty}\|y\|_{\varphi}^{R}+\|x\|_{\varphi}^{R}\|y\|_{\infty} .
\end{aligned}
$$

Hence, we can apply Lemma 4.1. Thus we get the main inequality (3). This completes the proof.

6. In this part, let $\lambda=\left(\lambda_{k}\right)$ be an increasing sequence of positive 
numbers such that $\sum_{k=1}^{\infty} \lambda_{k}^{-1}=\infty$. Denote by $\pi_{w}$ any finite non-ordered system of non-overlapping intervals $\left[a_{k}, b_{k}\right] \subset[a, b], k=1,2, \ldots, n$.

For $x \in X$ let

The number

$$
\sigma_{\lambda}\left(x, \pi_{w}\right)=\sum_{k=1}^{n} \frac{\left|x\left(b_{k}\right)-x\left(a_{k}\right)\right|}{\lambda_{k}} .
$$

$$
w_{\lambda}(x)=\sup \sigma_{\lambda}\left(x, \pi_{w}\right),
$$

where supremum is taken over all $\pi_{w}$, is called the $\lambda$-variation of $x$. The class $W_{\lambda}$ of all functions $x \in X$ with a finite $\lambda$-variation forms a Banach space if we define the norm of $x$ as $w_{\lambda}(x)$. This is equivalent to the definition given by D. WATERMAN in [9], [10]. Waterman gave interesting applications of functions of this kind to the theory of Fourier series, and his idea of using $\lambda$-variation has inspired further research.

Theorem 4. The space $W_{\lambda}$ with the norms

$$
|x|=\|x\|_{\infty}+w_{\lambda}(x) \text { or }|x|=2 \lambda_{1} w_{\lambda}(x)
$$

is a normed Banach algebra.

Proof. By application of Lemma 4.1 the proof follows immediately from the inequalities

and

$$
\|x\|_{\infty} \leqslant \lambda_{1} w_{\lambda}(x) \text { for } x \in W_{\lambda}
$$

$$
\begin{aligned}
& \sum_{k=1}^{n} \frac{\left|x\left(b_{k}\right) y\left(b_{k}\right)-x\left(a_{k}\right) y\left(a_{k}\right)\right|}{\lambda_{k}} \leqslant \sum_{k=1}^{n} \frac{\left|x\left(b_{k}\right)\left[y\left(b_{k}\right)-y\left(a_{k}\right)\right]\right|}{\lambda_{k}}+ \\
& +\sum_{k=1}^{n} \frac{\left|y\left(a_{k}\right)\left[x\left(b_{k}\right)-x\left(a_{k}\right)\right]\right|}{\lambda_{k}} \leqslant\|x\|_{\infty} w_{\lambda}(y)+\|y\|_{\infty} w_{\lambda}(x),
\end{aligned}
$$

i.e.

$$
w_{\lambda}(x y) \leqslant\|x\|_{\infty} w_{\lambda}(y)+\|y\|_{\infty} w_{\lambda}(x) .
$$

Remark. Let $\mathfrak{X}$ be a one of the three spaces $V_{\psi}^{*}, R_{\varphi}^{*}$ or $W_{\lambda}$. Since the convergence in each of them implies the uniform convergence on $[a, b]$ it follows that the space $C \mathfrak{X}=\mathfrak{X} \cap C[a, b]$ is a closed subspace in $\mathfrak{X}$ and for $C \mathfrak{X}$ Theorems $1-4$ can be repeated. 


\section{References}

[1] Bullen, P.S.: An inequality for variations. Amer. Math. Monthly 90, 560 (1983).

[2] Clavert, J.: Some generalizations of Opial's inequality. Proc. Amer. Math. Soc. 18, $72-75$ (1967).

[3] Cybertowicz, Z., Matuszewska, W.: Functions of bounded generalized variations. Comment. Math. 20, 29-52 (1977).

[4] LEŚNIEWICZ, R., ORLICZ, W.: On generalized variations (II). Studia Math. 45, $71-109$ (1973).

[5] Mrtrinovic, D.S.: Analytic Inequalities. Berlin-Heidelberg-New York: Springer. 1970.

[6] Musielak, J., Orlicz, W.: On generalized variations (I). Studia Math. 18, $11-41(1959)$.

[7] Musielak, J., ORlicz, W.: On modular spaces. Studia Math. 18, 49-65 (1959).

[8] Russell, A. M.: A commutative Banach algebra of functions of bounded variation. Amer. Math. Monthly 87, 39-40 (1980).

[9] WATERMAN, D.: On convergence of Fourier series of functions of generalized bounded variation. Studia Math. 44, 107-117 (1972).

[10] Waterman, D.: On A-bounded variation. Studia Math. 57, 33-45 (1976).

[11] WIDder, D. V.: The Laplace Transform. Princeton: University Press. 1946.

Lech Maligranda and Wladyslaw Orlicz

Institute of Mathematics Polish Academy of Sciences

Mielżyńskiego $27 / 29$

61-725 Poznań, Poland 